Received: January 25, 2021 / Accepted: March 24, 2021 / Published online: July 2, 2021 The (C) Author(s) 2021. This article is published with open access at Academia Analitica

ORIGINAL SCIENTIFIC PAPER

UDC: $111: 16: 81$

\title{
Logical Identity: a Holistic Approach
}

\author{
Nijaz Ibrulj ${ }^{1}$
}

\begin{abstract}
It is my intention in this article to present some consequences of Quine's thesis on the dependence of ontology on ideology (Quine, 1980), seeking an argument for my own thesis on the dependence (theoretical) existence of entities on identity type or ontology dependence on logic and language.If Quine's thesis is correct, then we can expand the resolution of this conclusion and say that ontology depends on the identity or on identification of the "identity criteria for conceptual schemes" (Davidson, 2001) which is constructed in the theory. Consequently I will speak about types of identity which adapts choice of ontology and of which depends ontology of a theory. Here I want to connect the different types of use of the term identity in Aristotle's writings and the different types of predications that are based on them with the concept of identity as the equivalence of symbols in modern logic. I want to reinterpret Quine's statement: "There is no entity without identity " in the form of imlication "What (kind of) identity such (kind of ) entity."
\end{abstract}

Key words: logic, language, ontology, epistemology, identity, holism,

\footnotetext{
${ }^{1}$ N.Ibrulj

University of Sarajevo, Faculty of Philosophy, Dept.of Philosophy

Franje Račkog 1, 71000 Sarajevo, Bosnia and Herzegovina

nijaz.ibrulj@ff.unsa.ba
} 


\section{Introduction}

In Quine's (1980) version of the philosophical explanation of how world, language and thought work together is established the chain of dependency: ontology of a theory depends on the language; the language of the theory depends on the conceptual scheme of language users; the conceptual scheme of the language users depends on the idioms of identity and quantification; the idioms depends on the culture in which are adopted by the natural language; the culture is dependent on psychogenetic and ontogenetic roots (Quine, 1976) of each individual. Every individual has therefore his own conceptual scheme that can be fully utilized only within a culture that shares the same ontology and the same language. A theory expressed in one particular language is not fully translatable into another language due to differences of idioms of identity and quantification ("indeterminacy of translation"), that is, and because of the ontology ("ontological relativity") or because of different inclinations of a society and a culture towards the attribution of property of existence to objects ("inscrutability of reference").Question about retrieval "objective reference" with Quine, according to Peter F.Strawson, begins with eleboration of theory and language and ends by decline in mentalism, into discovering of "psychological mechanisms" who remain in the background (Strawson, 2000, p.124).

This is a simplified scheme exposed relativistic and pragmatic foundation of knowledge - the holism of knowledge - which form from perceptual and rational blocks who epistemologically set the relation between world, thought, and language, and it is here given in general characters. If this scheme paraphrased in terms in which the world, thought and language appear directly one to other, then a chain of dependence looks somewhat different: ontological status of physical object depends on ostensive (re)actions of agents and singular parts of speech; singular parts of language depend on the idioms of identification and quantification of the language penetrated from conceptual scheme; the range of singular and existential quantifiers depends of position or level at which there is a physical existence of the object in the hierarchy of cultural facts (as Quine said "from atom to Homeric Gods "), that is depends on what is in one culture takes as physical and what as a nonphysical object; types of cultural facts depend on whether their roots predominantly ontogenetic (as part of perceptual psychology) or the dominant psychogenic (as part of mental ontogenesis).

By carefully reading the above paragraph will not miss the fact that what of the general scheme is not paraphrased in the particular is an expression of "idioms of 
identity". So far we have mainly followed the stream explanations given by the American philosopher Willard Van Orman Quine. His version of holism, as opposed to say, of those Donald Davidson, which is offered in the article Reality Without References (Davidson, 1977 In: Davidson, 1984), is reduced on the scientific theory and the conceptual scheme. The theory in Quine's kind of holism of knowledge has, as in Wittgenstein, internal and the outer limits: external border is the experience, but internal is defined by the structure of the conceptual scheme, what is the logical core theory. The subject of the judgement of experience are not individual proposition of a theory, but the whole theory all the way to its logical center, because the theory actually is a conceptual hierarchy made of ontological and linguistic hierarchy that is formed from the ontological and linguistic particulars and universals with different levels of generality. The theory is actually "equilibrium" of propositions (Quine, 1980, p. 43) who has relation with experience. This equilibrium that exists in theory could be thought of as the equilibrium of identity with different types of identification of identity. It is a suggestive thought of this text.

Knowledge, in Quine's theory, is doubly dependent: from ontology and from ideology. The same experience, the same ontology, in the conceptual scheme of another person may not be equally judged (Quine, 1980, p.10). The theory depends on the choice of ontology since the "one's ontology is fundamental to the conceptual scheme by which he interprets all experiences "(Quine, 1980, p.10), and on what are referential possibilities of language. Thus, Quine talks about inscrutability of reference, about indeterminacy of translation and about ontological relativity (Quine, 1960).

The holism of identity - an idea that I want to defend here - initially is , like the holism of knowledge, in reltion with the Wittgenstein's idea about the "language game" or the "life form" that contains in itself the network of analog reactions of mental, physical, linguistic, and social acts (Wittgenstein, 1948, In: Wittgenstein, 1960). The difference is that what would a developed theory of holism of identity what here suggest - which should be viewed identity as a network of parallel distributed reaction of identification, physical reactions, language reactions, reactions stimulated by external impact, reactions stimulated by social influences, reactions produced by ontology of first-person, reactions stimulated by ontology third person, reactions produced by social ontology in general and reactions produced by sequences or stereotypes of logical identity. Such an interpretation of identity was closer to Quine's later thinking in his book Philosophy of Logic as it is estimated that the substitutive interpretation of identity is more appropriate 
(Quine, 1970, pp.47-60) than the objects inteipretation of identity, as Quine strongly expressed in the book From Stimulus to Science (1995, p.91)

On the basis of Quine's slogan "No entity without identity", and on the ground that the entity for a theory that what the theory believes "what is" and what sets as existent (positum), whether it is about the Homeric gods or about physical objects, and on the grounds that the difference is only one of degree of belief in their existence, it would be possible to offer a consequential slogan: "How much identity that much of existence", i.e.: "What (kind of) identity such (kind of) entity", introducing the argumentation that it is not just about differentiated ontology or ontological hierarchy but about the differentiated concept of identity and about differentiated use of the sign of identity.

If it is necessary to get closer to the idea that I represent, and that can always open the possibility for new derivations, it must be characterized so that it becomes clear what is different from other ideas in the same area. Therefore, it is good to say the following directly: the holism of identity is the idea o of distinguishing interactive degrees identifications of identities and hierarchies of representation that intertwine or distribute in parallel in one functionalist definition or in the function of unambiguity that connects them as a whole. In other words, If Quine's claim holds that the ontological difference is a matter of theory and its degree of belief in the existence of entities which are engaged, then one can also argue that identity, which is within the theory attributed to these entities in the process of creation of knowledge about them, dependent on whether the identity form of the relationship between the entities to be one essence (substantial identity-unity), from the standpoint of a quality that entities have independently of the essence that is different (qualitative identity-similarity) or from the standpoint of quantity of a property which they possess (quantitative identity-equality). Of course, this differentiation in terms of ontology can be expressed as the distinction degree of ontological generality which attaches or ascribe to entities in one theory (in which otherwise there is nothing singular) and at the same time is depending on the hierarchy and the capacity of logical forms that are filled with atomar propositions about given entities, and on the hierarchy of languages which are used for scoring identification of identity in the above theory.

My intention in this article is to outline some consequences of Quine thesis about depending ontology from ideology (Quine, 1980), or depending the entity from identity, or of dependency of ontology from the language. If Quine's thesis is correct, then we can expand the resolution of this conclusion and say that ontology depends on the identity or identification of the "identity criteria for conceptual 
schemes "(Davidson, 2001, p.184) which is constructed in theory. This means that we want to talk about different types of identity which adapts choice of ontology and of which depends ontology of a theory. I would like to remind here of the distinction between types of identities to which Aristotle already said (M.1021a10-15) something when he distinguished "identity of things" based on the network of concepts and words that name them and that are predicated to them (1) as homogenity / to be identical by essence, to be in same genus, (2) as specificaly identical / to be similar by species or by specific quality, and (3) as numericaly idnetical / equal, ie: synonymous identity of things, homonymous identity of things and paronymous identity of things (Aristotle, Categories.1a1-1a20). If, therefore, Quine's statement: "There is no entity without identity" is valid (Quine, 1969, p.27), then it could also be valid the claim "What identity such entity"

Quine spoke about this dependence as about dependence on the language of conceptual schema or idioms of identity and quantification, but under that is mostly or largely thought of idioms of quantification, or quantification of variable which dominates our entire ontology. Idioms of variable quantification, quantificational words or "variables of quantification" according to Quine (1980, p.12) are above the whole of our ontology: the terms "every", "some" and "non" are the only way our entry into "ontological commitment", or into ontological choice of a theory to which we commit from the periphery to the center, or how now I want to say, at all levels of identification of identity. These idioms in fact stand in the grounds of Rudolf Carnap statement that " to be mean to be part of the system "(Carnap, 1956, p.207) (Carnap thought of linguistic system or linguistic framework), and then stand in center Quine claims: "To be is to be the value of the variable" (Quine, 1980, 15). But this way of existence of objects is strongly complicated by the logical connectives within the logical calculus or within the calculus of propositions. Idioms of quantification demonstrates the scope of one fact is taken as an objective ("object bound variable "), and not what kind of existence is ascribed to the fact. This can be done, in my opinion, only by the idioms identification of identity by which two things are identified as "identical" because they have the same essence (exist in the same genus) and common name, or because they are only named with the same name because of one property they share (or in which they participate), or because the name of one thing is derived from the name of another thing.

The idioms of identity identification are what we want to point out here. Thereby are important reasons why these idioms, peripheral and centric, harmonize and build equilibrium of identity in different types of predication. In particular I want to point out the following: the type of identity or type of identity identification is in the 
direct connection with the kind of existence that an object has before it is ascribed to him or modified or only confirmed within one theory. And that means that we adopt the kind of realism that according to John R. Searl (1998, p.11) objects have "by default". This also means that we need to rethink Aristotle's opinion in a new way that the identity is the relationship between things $(\omega \nu)$ and not between words "without reality". This disrupts the harmony of relativism which the relationship between the world, language and thought brings Quine and for the initial ontology is taken the one that lets something be before we say by our theory that it is so-andso.

\section{The Use of a Sign of Identity}

Ludwig Wittgenstein in his two main works, stronger than Frege or Russell, started an interest in research of the world-language-thought relations, actually carried out a different use of the sign of identity..

In the Tractatus Logico-Philosophicus Wittgenstein (1922 In: Wittgenstein, 1960) try to analyze this relation by identifying and representating it as a structural unification of the world and language by a special using sign of identity as a sign for the equivalence of two sets: a set of the WORLD and a set of the LANGUAGE in the form of $\{\mathrm{W}\}=\{\mathrm{L}\}$, where it is about positional classification of the facts that make up the ontology of the set $\{\mathrm{W}\}$ and the facts that make ontology of the set $\{\mathrm{L}\}$. In doing so, their identity is postulated as a result of the mathematical criteria of equalization of the given in the method of mutual mapping of the both sides, which is due to the philosophy from the works of Georg Cantor over Gottlob Frege to Wittgenstein. Therefore, in Tractatus the set $\{\mathrm{L}\}$ is the image of the set $\{\mathrm{W}\}$ because the atomic proposition $\{\mathrm{p}\}$ is a image of the atomic fact $\{\mathrm{w}\}$.

In Logische Untersuchungen Wittgenstein (1948 In: Wittgenstein, 1960) changes the sense of using the sign of identity seeking semantic unification of the world and the language in the form $\{\mathrm{W}=\mathrm{L}\}$ which is analog form $\{\mathrm{L}=\mathrm{W}\}$ and to any other which is derived from this form, where the one set consists of the one holistic language game $(\mathrm{l}=\mathrm{w})$ or one form of life $(\mathrm{w}=\mathrm{l})$, which contains a network of mental, physical and linguistic reactions as one set of facts which are in correspondence or are in analogy. The criterion of identity in this theory is given in functional definition or in use of the idiom of identification for each language game particulary. It is therefore not possible to know in advance the meaning of words in a language game as it is not possible in advance to determine its ontology: it depends on the type of stimulation, which can be physical, mental, verbal, or social. If in one language 
game are set all of its elements, then it is meaningful unification of all reactions. If words have no meaning or if they can not have it with regard to the criteria of identification that dominates in linguistic game, then there is no objects to which can be attributed.

However Quine's slogan "No entity without identity" (1969, p.27), can't be converted if the term entity thinks something that has existence in space and time. Conversion in this case does not apply. This is precisely the reason why is possible to accept his claim about an ontological positum: something that is the physical object and something that is Hermes (one of the Homeric gods) can equally be the object of a theory, and vary only the degree of existence that they are in this theory is attributed, and not belonging to them by itself. This means that the identification of their existence, be it external or internal due to the holism of the mental, regardless of the degrees of state of things and processes and the degrees of mental states of things and processes, is dependent on the conceptual scheme and that their existence within the theory is further dependent from the theory of language which represents the achieved identification. Attributing (ascribing) the meaning to the words is in direct relation to attributing the existence of objects that are identified.

Let's try to repeat Quine's Gedankenexperiment wit the rabbit and the identification of the identity of "rabbit stages" (Quine, 1960, pp.26-31), now in the second "type of attribution". Description of movement of the rabbit in one space made in the vocabular of natural language differs from the algorithm of movement of the rabbit which is written in the vocabular of mathematical language. It is important that in the conceptual scheme which works in the natural language of an object such as a "rabbit" (lat. Lepus) identifies with "rodent that belongs to the family of mammals characterized by the broken cleft upper lip, long hind legs, a short tail and long ears "or, in the child's epistemology, as "sweet-with-large-ears-fast-hairy-hot-animal" which is "not called squirrel "than just named by word " rabbit ". In mathematical description of a natural state of things, or moving of the rabbit, object identification is not done through its definition than is done by description one of his spatiotemporal properties without specifying what is the object or what is characteristic of movement of the rabbit. The question is how an object behaves in space and time given a characteristic which is desired to be identified at various time sequences. What inside a culture that covers the space and time where this description is done means rabbit paw - it does not matter, as it does not matter in what kind of animal is one rabbit and if it's the same kind in which falls squirrel nor which specific differences exists. Even less is essential that the Latin name for the wolf is Lupus. 
At this point, we can go through which is set Gottlob Frege (1918 In Patzig, 2008) in the article Über Sinn und Bedeutung wondering whether the identity is the relations what is claimed / attributed (by a theory which contains unambiguous symbolic language) or what exists by itself. Frege expressed this question of whether the identity relations is relation between signs which designate some objects or relations between the objects themselves. Graphic difference between "a = $\mathrm{a} "$ and " $\mathrm{a}=\mathrm{b}$ " was enough to suggest to Frege that without danger to the truth (truth value of the statements) the same thought content (same thought) be represented in different ways in the same artificial (symbolic) language. But the question that might arise if moved away from Frege and come closer to Quine's proximity is: is there " $\mathrm{a}$ " in the phrase "a $=\mathrm{a}$ " more or less existence or the same ontological status than it has "b" in the expressions "a $=b "$ ?

By this we are not far from the road that went Strawson (1990, p.17) when asked what is ontologically primary, "a" or "b", and concluded that "a" is primary if it contains in itself " $\mathrm{b}$ ". It is an analytical question, about the hierarchy of languages and complexity of symbols that is to Strawson came through Carnap. My question concerns the differentiated ontology and differentiated identification of identity. Whether in the same theory can be entity with different ontological status at different levels of theory, and hence hierarchy of ontological generality, and whether this means the hierarchy of existence objects or hierarchy of identification of existence of objects? Secondly, what does it mean for the theory itself, for idioms quantity and identification? Maybe it is good at this point to recall the Wittgenstein's intellectual effort and ask whether by the type of identity (or the type of attribution of meaning, and then existence) we adjusts structural or semantic unification of the ontologies and language?

It is necessary, however, to rethink what follows from such relationship to the ontological and epistemological assumptions of the theory. Quine considers entities of the scientific theory as cultural facts that differ only in degree of belief in its existence and not by type of its identity and existence: physical objects and the Homeric gods are positums (Quine, 1980, p.44) entering the theory on the basis of belief or on the basis of their existence attributed to them in a cultural framework. Well, if so, then we would have the right to bring the level or degree of existence in correlation with the level or degree of identification and to say: "What an entity such an identity." Or: "As much there is identity, so much there is entity". To remind on the other way to a notion here I defend is the following: if the object of thought differ in degree belief in their existence, the degree of their positioning in a system of beliefs on which this positioning rest, then there is the mental coherence 
of knowledge of these objects differs by the degree of identity identification or by type of identity that is epistemologically formed.

\section{Ontological Status and Identification of Identity}

Quine's distinction between ontology and ideology of the one science theory (Quine, 1980, p.131) is an integral part of the thesis that the world is dependent on the conceptual schema of language, which is filled by it. Ontology is dependent on the particular culture or ontological facts are cultural facts. That what within a culture is taken as existing, no matter how existence has and how much depends on the sensory stimulation, works in a theory ("theory based on one specific culture ") as an object about which is constructed a theory which is made up from the center (logic) and peripherals (experience). The culture, as for example was ancient culture had in its ontology included the immortal Olympian gods ( $\dot{\alpha} \vartheta \alpha^{\prime} \nu \alpha \tau o \iota$ $\left.\vartheta \varepsilon \circ \iota\right)$ and various mythical creatures as existing, as that "what is" (what exists) next to people, things, animals, plants and heavenly bodies.

But what stands in relation to all these cultural facts as a scientific paradigm or (at one time) as rationally acceptable ideology of science is a theory that gives physics as a science of nature and that speaks about self-created matter, for which exist atoms and electrons, forces and fields of forces, movements and rest, and for which without contradiction can't speak about immaterial beings as space-time phenomena in terms of describing things (the language about things). On the scientific paradigm, which lovers of ancient culture may or may not must adopt as true or as rationally acceptable, which can accept or reject, is based one conviction which is called atheistic and located in all monotheistic cultures as well as cultural facts for whose argument in the language about things is the most rational evidence, or whose idioms identification identity provide the highest level uniformity (one-meaningness).

Different cultures with different ontologies or share or not share, accept or do not accept one physics: the one to which the educated people come at one time. Within one, second and third or fourth culture it is known exactly what is a physical object and what are the physical properties, and what is the language about things in which explicates this ontology, we know what are the mental objects and their properties, what is the language in which this ontology explicates, and we know what are the objects of beliefs and their properties and what is the language of the theory in which this ontology explicates. It can not be unambiguously theory as the set "periphery / experience-plus-center / logic" applied to all cultural facts although all ontological states can be declared as cultural facts. 
Within a culture strongly dominated by monotheistic belief, statements containing a supernatural ontology cannot be verified individually or all together based on the experience of the periphery shared by physical material objects with atomic structure and the force of gravity. They are verified in relation to experience beliefs of the entire believing community, in relation to holism of the mental within which the mental states of belief and hope appear in types of representation or types of statements "I believe that___, "I hope that__," and what identifies these mental states as real or as existing forms of one ontology of belief rather than a phenomenology of things. The question of whether words are rigid signifiers in all "possible worlds", initiated by Saul Kripke (1972), could also arise in the form: whether the identification of identity and contradiction, which is related to the identification of truth and falsity of statements, performs according to the same rigid logical principles in all "possible conceptual schemes", phenomenological and non-phenomenological, or maybe we have one principle for one ontology and another for other?

What I want to suggest here is this: primary or particular, not global, ontologies of different theories are not different because the cultures that produce them are different but because the identification framework of the theory, and then the ontological status of objects, is complex regardless of culture which produces it. The ideological or identification status of objects that is ascribed to objects in one theory depends on the dominant type of identity that the theory constructs on one of its levels. For example, the hypothetical level of a theory is not equal to its analytical or synthetic or conclusive level. Idioms of identity identification depend on culture and language, but their function is universal for every culture: they serve to discriminate sensory and perceptual objects, large and small forms, current and frequency configurations, short-term and long-term procedural activities, mental discrimination of object classes, formalized class discrimination of classes, etc. They permeate all hierarchies of the perceptual and mental process of identification, beginning at lower levels and ending at higher cognitive levels (Kosslyn, 1995).

The identification area of a theory provides identification status objects theory by being in that area or at that level, which may be hypothetical, or analytical, or synthetic, provides criteria identity that sets the basis for the treatment of identity as superior / conditional identity, categorical / unconditioned identity, analytical / deductive identity, synthetic / multyconditional identities and so on. The identification status by object gets suddenly highly theoretical criterion of identity which satisfies the uniformity of the function which is formed at that level and 
involving all parts of the conceptual content that semantically and structurally adjusted to the truth-conditions values imposed by the criterion of identity.

Within each culture there are entities to which are attributed existence as a spacetime (physical), as conceptual (logical), as well as mental (psychological), as well as linguistic (verbal). In regarding the nature of existence or level of ontological context that is attributed to the objects there is formed or constructed theories in language which belongs to it by the context in which it belongs. So, what by itself suggested at this level is that you should not seen relationship between entity and identity within the theory in the scheme of "one culture - one ontology "(one entity one identity), but can speak o complex ontological context in which it belongs more or less an object of the theory, which can be more or less more objectively works. The ontology of a theory cannot be in collision with the logical center of that theory, i.e. with what there is a logical instrument of grounding and justification for. One culture produces a multitude of ontologies and languages that belong to them contextually. There is, however, a different level of identity that can be formulated or achieved with such an ontology and with such a vocabulary. Identity is a whole, something that a theory should achieve by adjusting semantic and structural sequences, which, if scientific, stand within logical stereotypes composed of if-then sequences. The criterion of identity is what Davidson refers to as the "criterion of translation" for conceptual schemes (2001, p.184), and what can be characterized as the criterion of adjusting the structural and semantic levels of identification.

Let's remember: Quine did an ontology of one theory dependent on the ideology of that theory. His slogan "No entity without identity" may mean this:" There is no ontology (of a theory) without ideology (just this theory) ". This actually expresses that the ontology depends on the language of the theory in which it appears. That could means: "There is no ontology without ideology". However, I introduced as a counterweight the slogan "What ideology such an ontology", i.e. "What identity such an entity".

Let's try now to make own Gedankenexperiment that would be helpful to the arguments of this statement. We can, for example, accept the ontological context of the KAPPA books of Homer Odyssey as part of a separate theory arose within a culture and distinguish it from the ontological context LAMBDA books. Inside KAPPA book can be considered a cultural fact or ontological positum " beautiful-

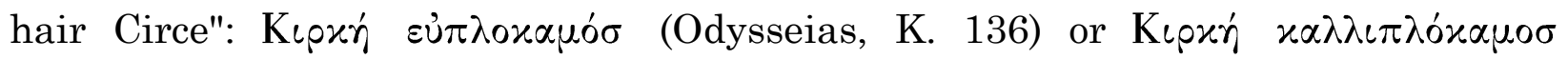
(Odysseias, K. 220), or "herbal-healing Circe" dealing with herbs and prepared a wicked poisons to make men forget their homes; we can accept and that she is able to turn with the stroke of a stick sailors in pigs. But in this context and at the 
account of such an ontology we can not in its operational conceptual scheme give to Circe the same ontological status which have eg. " beautiful-haired graduate pharmacist employed in pharmacy 'Old Town' in Sarajevo " and / in " plavolasa diplomarana farmacevtkinja, ki dela v 'Lekarni' na Miklošičevi ulici v Ljubljani" (regardless of the different languages in which these two ontology, as opposed to those of Homer, appear) although dealing with herbs, but none of them is able to perform miracles with magical bat nor is able to make magic drinks of which man forgets his country. Also we can not identify the meaning of words which in the book

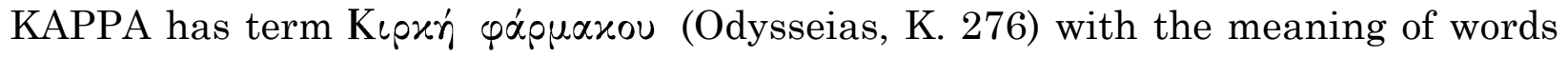
"apotekarka Zlata" or "farmacevtkinja Mojca". We can do ontological gradations within the KAPPA books and say that the existence of pigs has higher degree of ontological existence then magician and herbs-collector Circe, but we can not graded whole ontological context in relation to the ontological context LAMBDA books, where Odysseus descends to the Underworld and encounter dead souls waiting to drink the blood of sacrificed animals, and to tell the truth, or ontological status of the soul of the prophet Tiresias ftom Teba that Odysseus speaks the future (Odysseias, L., pp.100-135). Both of these books, KAPPA and LAMBDA, belong to the superior context of Odysseias where there are 24 parallel ontological context each with its own ontology or with varying degrees of identity identification (spoken by my terms) or ideology (in Quine terms).

\section{Logical Identity and Its Semantical Differences}

The type of identification of identity speaks about the kind of existence of entities. Identity is primarily type of identification, the way the parts of thought content equate according to their semantic function participating in the formation of a statement as a function of unambiguity that has a truth value because it has by itself a (logical) law or, as it is called Donald Davidson "criterion of identity for conceptual schemes". At the same time the identity is a kind of representation of this unambiguity or the function that have not only semantic, but also structural competence or "fulfillment" ("satisfaction") (Tarski, 1956, p.189). One simple thought content can be represented by a complex symbol, for example by double negation, while a complex content can be represented by a simple symbol.

Now let's look again at what is offered by Aristotle in differentiated concept of identity exposed in Metaphysics (M.1021 a 10). If we want to identify things ( $\tilde{\omega} v)$ as identical, then it can be done in three ways 


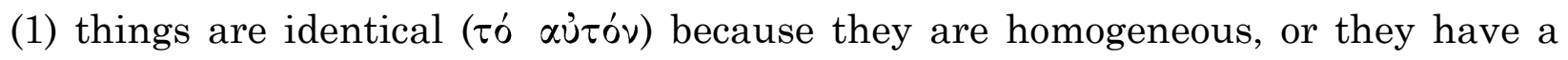

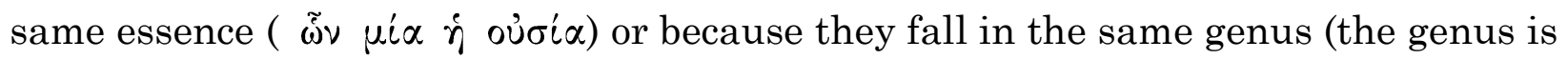
the essence of things);

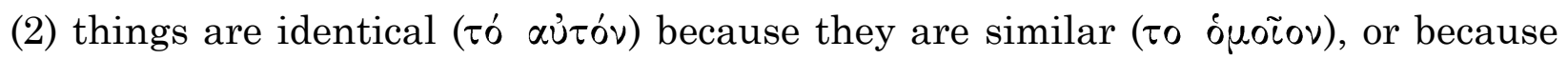
they have same quality of essence ( $\left.\omega \nu \eta \pi \sigma^{\prime} \delta^{\prime} \eta \sigma \alpha i \alpha\right)$; and

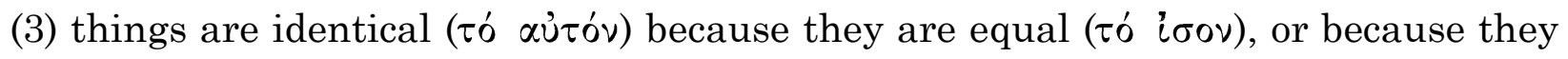

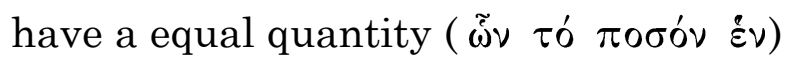

Let's try now to connect these three ways of identifying identity distinguishing between their linguistic-grammatical and logical realization through their triple kind of predication based on synonymy, homonymy and paronymy, about which Aristotle speaks in the Categories (Aristotle, K. 1a 1 - la 15). In the case of (1) if things have one essence then they have (a) a common name (óvou $\alpha$ rocvóv) and (b) identical concept of essence (definition) marked with the name ( $\delta \delta \varepsilon_{\varepsilon}^{\prime} \varkappa \alpha \tau \alpha \dot{\alpha} \tau o u ́ v o \mu \alpha$

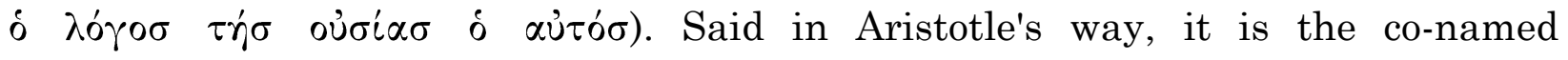

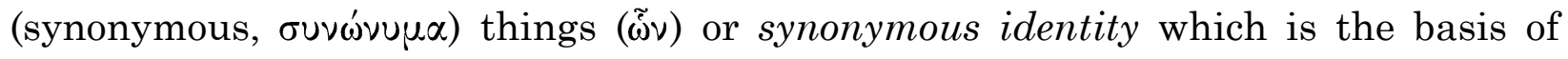
synonymous predication and which necessarily connects the subject and predicate. In this context, we should name things by common (and not same) name because

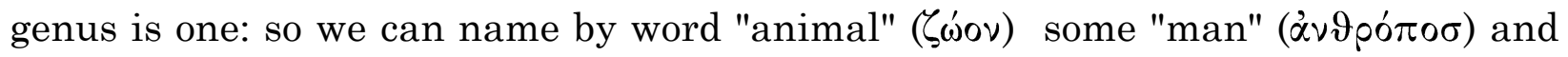
some "ox" (ßoũ $\sigma)$ because they fall under same genus which is essence attributed to them by deffiniton. So if we want to say by what man and ox are identical, then we can say that they both are "animal". This is a remote or generical logical notion of identity. If we want to prove this definition and state why the common name "animal" appears in the definition of man and ox, then we will list the properties of the animals that belong to man and ox to the same extent. This notion of identity is about logical or analytical identity.

In the case of (2) if things have one quality, then they have (a) only the common name (óvoux yóvov xocvóv) and (b) different concept (deffinition) marked with the

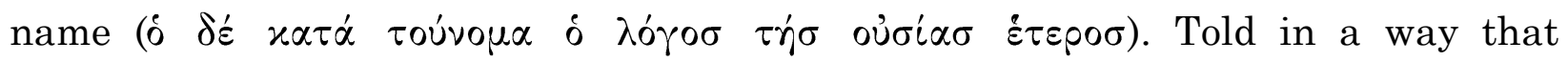
Aristotle spoke on it in Categories it's about the same-named (homonymous,

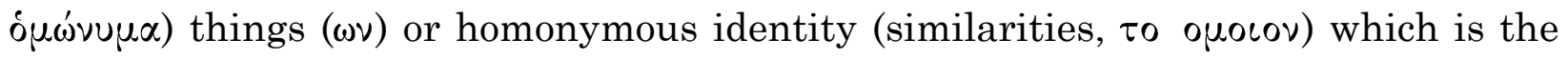
basis of homonymous predication or accidental connection between subject and predicate. The homonymous predication is synthetic, ambiguous, because it does not follow from the same definition but from accidental belonging of two or more predicates to one subject. Things can only be called a common name, but they do not have the same definition as it does not belong to the same genus and have not the same essence but just same quality. One single "man" on the street and "image of 
man" at the Greek vase can be named by a common name as "animal", but definition or the concept of "man" is different from the definition or the concept of "painted man".

In the case of (3) if things have analogical name, a thing is named by analogy to some othar thing. Then the name of one thing derives from the name of another thing: the name for a "grammarian" is derived from the name "grammar". In the way Aristotle said on this in the Categories it's about paronymous things ( $\omega \nu)$ or about things named by the name of its same quality or things having paronymous identity (analogy of quality) or on paronymous identity in paronymous predication .

Now we go back again to what is sed by Frege in the article Über Sinn und Bedeutung. The difference between " $\mathrm{a}=\mathrm{a}$ " and " $\mathrm{a}=\mathrm{b}$ " is not only difference in the type of representation that says that sign " $b$ " is an equivocation or homonym or another name for "a", or that "b" is a different way of existence of linguistic entities "a" as the sign " 5 " is another way of the existence of linguistic entities " $2+3$ ". Rather this difference either indicates a difference in the ontological status of the entities that are brought into the identity relation or indicates a difference in the types of identification of identity attributed to the same entity. It is shown that the named entity does not allow denoting with only one set of characters or that it can be applied to it only with one criterion of conceptual scheme, or only with one criterion of translation, within which one conceptual content is identical only to itself (substantial identity), but that he himself, or one of his properties which characterizes him in definition and in which he can be transformed into a definition, may be denoted by a series of signs formed in a single function of unambiguity. Cognitive conversion or cognitive synonymy is what Frege established as a possibility of his Begriffsschrift.

Here, then, it is not a question of whether an object or entity exists or does not exist, but in what way it is possible for it to exist for us as identical in all of its phases, in the physical, in the mental and linguistic complex which we form. If "b" is a common name for "a" and "b" or if "a" and "b" are two different signs for the same entity, then the difference is in what entities are identified as identical. If the relation "a $=$ $a "$ and the relation "b $=b "$ are contained in the relation "a $=b "$, then nothing in the signs thus written allows a transition to the relation "a $=b "$, unless there is something third by which both characters are connected as his names and which gives them an identity. In Aristotle's syllogistics it is not possible to prove that the relation " $\mathrm{a}=\mathrm{b}$ " is valid as true if there is no common name / sign "c" for which it is valid: "a $=\mathrm{c}$ " and " $\mathrm{b}=\mathrm{c}$ ". In Aristotle's syllogistics it is not possible to prove that is true as true relation " $\mathrm{a}=\mathrm{b}$ " if there is a common name / mark "c" for which applies: 
$\mathrm{a}=\mathrm{b} "$ and $" \mathrm{~b}=\mathrm{c}$. Because of that, in Aristotle there is a difference between definitions: $\delta \rho i \sigma \mu \circ \sigma(\mathrm{a}=\mathrm{b}, \mathrm{b}=\mathrm{c}, \mathrm{c}=\mathrm{d})$ and evidence: $\dot{\alpha} \pi \circ \delta \varepsilon i \xi_{\iota} \sigma(\mathrm{a}=\mathrm{b}, \mathrm{b}=\mathrm{c}$, then $\mathrm{a}$ $=\mathrm{c})$.

Frege took this relation as equivalent of its designations of content: "a" and "b" are different linguistic ways of conceptual existence of the same object, different way of giving the same conceptual content represented by these signs. For Frege there is the same identity in the expressions: "Venus = Venus", "Venus = die Morgenstern", "Venus = die Abendstern", "die Morgenstern = die Abendstern" and all the other combinations that follow. But Frege's words and sentences linked not only with things and objects but also with concepts, with the sense of words and not just with their meaning. Thought for Frege is the sense of proposition and the way its linguistic givens or structures of thought (Gedankengefüge) (Frege, In Patzig, 1966, p.72). In the case of $\mathrm{a}=\mathrm{a}$ " we are talking about an analytical proposition and talking about the logical identity or equality of entities with themselves (substantial identity) which is valid regardless of the type of entity, whether they are imagined or real, whether their existence of this or that degree, be they entities or semientities. It is valid even when the whole reality would be reduced to the sign "a".

Let us now consider only the case "a $=\mathrm{b}$ ". In the traditional sense that it is about synthetic proposition. Let's see now, trying to connect traditional (Aristotle) and modern (Frege) interpretation of the notion of identity in some opportunities designed just as Gedanekenexperiment for this exercise. What are possible combinations or syntheses or just answers to the question of what is meant by this form of identity? Let's take into account those thoughts that are counterfeit and that we can only imagine without any real possibility to get confirmation in experience.

\section{a) Substantial identity (identity of the things)}

(1) the relation between entities: entity "a" and entity "b"

(2) the relation between the signs / names that refer to the same entity, between the sign "a" and the sign "b"

(3) the relation between the existence of the one entity in different time sequences

(4) the relation between the phases or sequences of one entity in space

(5) the relation between different types or levels of identification of one entities in the process 
(6) the relation between different levels of identification of the two entities

(7) of the relation between different parts of one logical content: subject "and" the predicate "b"

\section{b) The qualitative identity (similarity of the things)}

(8) the relation between the degree of identification of some of the same properties of one entity, ie. different levels of logical generality that attributed to one propety and different hierarchy of complexity of representation which the property represents

(9) the relation between the degree of the logical generality under which fall entities

(10) the relations between two ontological degree of generality, which has an entity in theory

(11) the relation between two linguistic degree of generality that apply to an entity

\section{c) Quantitative identity (equality of the quantity of things)}

(12) the relations between the different degrees of quantity of the same properties which posess one entity (quantity "a" of the property $\mathrm{D}$ of the entity $\mathrm{P}$ and quantity "b" of the property D of the entity P.

(13) the relations between the different marks of the same property for the one entity (mark "'" a "'" of the property "a" for the entity $\mathrm{P}$ and mark "'" $\mathrm{b}$ "' of the property "b" for the entity $\mathrm{P}$.

Here we let our imagination run wild to visually show a number of mental rotations that lie behind the idea of identity holism. At all levels or in any isolated type of identity identification it is obvious that all other types are present, in one way or another. In fact, in each individual possibility from (1) to (13) the ontological, logical and semantic types of identity identification are intertwined in parallel. These identity differentials clearly show the complexity and intertwining of types of identity identification. This is to say that identity is not a rigid analytical identity of an object with itself, nor is it a rigid or necessary affiliation of a predicate to a subject that is thought of as already present in the notion of the subject.

There is even a difference in the notion of necessity or rigidity, the one described by Saul Kripke introducing the phrase "a priori based on experience" (Kripke, 1972). In 
other words, the path to rigid conclusions in one theory is difficult, gradual, and it establishes a network of identity identification in language and metalanguage, in the first consequences and in later derivations. In addition, identity, like contradiction, must be re-identified each time (Ibrulj, 1999: 212) and therefore scientific theory, like everyday speech, is always open to degradation, reconstruction and interpretation.

Idioms of identification are truth functions, open statements which in addition depend in their ontological capacity on related variables or on the idiom of quantification that function in some language, also depend on the system or construction of the type of identity that is established at one theoretical level and which integrates and distributes subjects and predicative parts of statements by arranging logical matrices.

\section{Conclusion}

The strategy of this text was predetermined by the idea that not only does the ideology of a theory determine its ontology, but that the different types of identities of the theory contains as algorithmic blocks or stages of identification of parts of conceptual content, and which it uses as elements of a function of unambiguity that reaches objects of experience, determine the ontological status of objects theory, the type of entity and thus the context and language of the theory, that is, everything that enters into the complex notion of the truth value of the whole theory.

It was more than a seductive opening up opportunity to connect Aristotle's complex notion of identity or triangulation of identity as a logical-ontological-linguistic framework within which subjects and predicates are distributed and integrated in parallel at different levels by means of identification criteria acting through homonymous identity, synonymous identity and paronymic identity. It turned out that this difference that resides in the use of the sign of identity or in the ways in which the same is said should be used only in a certain direction and scope, or more as an illustration of the possibilities to which, as Aristotle himself says, "the same is

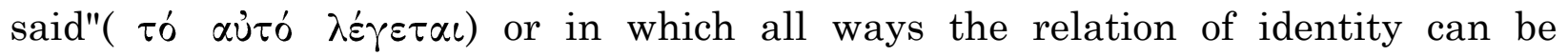
considered when thinking about the language-world-opinion structure which is exemplified or adjusted in one relation in a semantic and structural sense. 
Identity is a complex relation that emerges on a semantic and structural level, concerning logical, ontological and linguistic particulars and universals from which lay, religious, mythological or scientific theory is formulated o objects that can be called cultural facts, as Quine does, but for which in every culture there is an established stereotype of the existence they have and which is attributed to them. This stereotype depends on the type of identification that is arranged within the theory that is chosen for the type of objects that should appear in it. The theoretical paradigm or stereotype of identification does not arise from the discovery of an object, whatever its properties, but strongly influences whether the object will show itself in the light of theory as physical, rational, irrational or fantastic.

In contexts in which several objects of different identification criteria occur, it is possible to establish ontological discrimination by determining the ontological status of individual objects in relation to the cultural context from which they are positioned, in relation to the global conceptual scheme or global picture of the world (Davidson) shared by participants in a culture within which there is a linguistic division of labor (Putnam) but also knowledge o of the types of ontological status objects have in themselves.

The idea about the holism of identity is an idea that goes in favor of understanding a theory as a set of criteria of identity, each acting vertically, in the direction of synonymous or in the direction of logical relationships of belonging, inclusion and predication, as the relations of different levels of logical generality; horizontal direction or in the direction of reduplication names ending with deviant, what paronymic identity, and in a direction that operates with the same names or homonymy type of identity.

\section{References}

Aristotelis Organon I-II. (1948). Übersetzt und erleutert von Eugen Rolfes. Leipzig: Velix Meiner Verlag.

Aristotelis Metaphysica l-II (1978). In griechiesch-deutsch. In Übersetzung von Hermann Bonitz. Neu bearbeitete mit Einleitung und Kommentar von Hoerst Seidel. Hamburg: Velix Meiner Verlag.

Carnap, R. (1956). Meaning and Necessity. Chicago: The University of Chicago Press. 
Davidson, D. (1977). "Reality Without Reference", In Davidson, D (2001): Inquiries into Truth and Interpretation. Oxford: Oxford University Press.

Davidson, D. (1974). "On the Very Idea of a Conceptual Scheme", In Davidson, D (2001). Inquiries into Truth and Interpretation. Oxford: Oxford University Press.

Davidson, D. (2001a). Subjective, Intersubjective, Objective. Oxford: Clarendon Press.

Frege, G. (1966). "Gedankengefüge", In Patzig, G. (ed.). Gottlob Frege: Logische Untersuchungen. Gëttingen: Vandcnhoeck \& Ruprecht.

Frege, G. (2008). "Über Sinn und Bedeutung", In Patzig, G. (ed.). Funktion, Begriff, Bedeutung. Gëttingen: Vandenhoeck \& Ruprecht.

Homeri Odyssea. Edidit by Guilielmus Dindorf. Editio quinta corrector quam curvat C. Hentze. Pars I. ODYSSEAEI-XII. LIPSIAE in aedibus B.G. TEUBNERI. MDCCCXCI.

Ibrulj, N. (1999). Filozofija logike. Sarajevo: Sarajevo-Publishing.

Kosslyn, S. M. (1995). Image and Brain. Cambridge, Massachusetts, London: MIT Press.

Kripke, S. (1972). Naming and Necessity. Oxford: Clarendon Press.

Putnam, H. (1975). Philosophical Papers 2. London: Cambridge University Press. Quine, Willard Van Orman (1960). Word and Object. Cambridge, Massachusetts and London: MIT Press.

Quine, W. V. O. (1980). From a Logical Point of View. Second Edition. Cambridge, Massachusetts and London: Harvard University Press.

Quine, W. V. O. (1976). Die Wurzeln der Referenz. Frankfurt / Mein:Suhrkamp. (Quine, The Roots of Reference. La Salle, Illinois: Open Court Publishing Co.

Quine, W. V. O. (1970). Philosophy of Logic. New York: Prentice-Hall. 
Quine, W. V. O. (1995). From Stimulus to Science. Cambridge, Massachusetts and London: Harvard University Press.

Quine, W. V. O. (1969). Ontological Relativity and Others Essays. New York: Columbia University Press.

Searle, J. R. (1998). Minad, Language and Society. Philosophy in the Real World. New York: Basic Books.

Strawson, P. F. (2000). Entity and Identity and Other Essays. Oxford: Clarendon Press.

Strawson, P. F. (1990). Individuals. An Essay in Descriptive Metaphysics. London and New York: Routledge.

Tarski, A. (1956). Logic, Semantics, Mathematics. Oxford: Oxford University Press.

Wittgenstein, L. (1922). "Tractatus logico-philosophicus", In Wittgenstein, L.(1960):

SCHRIFTEN. Frankfurt am Main: Suhrkamp Verlag, pp.11-83.

Wittgenstein, L. (1948). "Philosophische Untersuchungen", In: Wittgenstein, L.(1960): SCHRIFTEN. Frankfurt am Main: Suhrkamp Verlag, pp.289-543. 